\title{
UNIFORMLY DISTRIBUTED MEASURES IN EUCLIDEAN SPACES
}

\author{
BERND KIRCHHEIM and DAVID PREISS
}

For every complete metric space $X$ there is, up to a constant multiple, at most one Borel regular measure $\mu$ over $X$ such that

$0<\mu(B(x, r))=\mu(B(y, r))<\infty \quad$ whenever $x, y \in X$ and $r \in(0, \infty)$;

where the symbol $B(x, r)$ stands for the closed ball with center in $x$ and radius $r$. In fact, the existence of such a measure imposes rather strong regularity conditions on the space $X$ itself (see [2], [7], [10]); in particular from [3] we know that, if $X$ is a bounded subset of $\mathrm{R}^{n}$, then it is contained in a sphere. In the case when $X \subseteq \mathrm{R}^{n}$, regularity results about $X$ turned out to be the most important tool in the study of densities of measures in $\mathrm{R}^{n}$ (see [9], or, for a more detailed but less complete account, [8]). One of our main results considerably improves several results from [9] by showing that any $X \subseteq \mathrm{R}^{n}$ is an analytic variety. Our proof is much simpler than those presented in [9], which enables us to give as an application a simple proof of a remarkable result of Marstrand [6] according to which the existence of non-trivial $s$-dimensional densities (of a measure in $\mathrm{R}^{n}$ ) implies that $s$ is an integer. Our results, however, do not seem to be strong enough to show the main result of [9] that measures having $s$-dimensional density are $s$ rectifiable, because they do not give any information about the behaviour of $X$ at infinity.

In addition to the analyticity result mentioned above, we also show that $X$ is an algebraic variety provided that it is bounded and obtain more precise results in the special cases $n=1$ and $n=2$.

For various reasons, including the political changes in Europe, this note has not been published for many years, although our main approach has become known and used in the literature. We would like to thank the Max Planck Institute for bringing us together and thus enabling us to recover the manuscript and prepare it for publication.

Received February 11, 1999. 
Under a measure $\mu$ over $\mathrm{R}^{n}$ we will understand a monotone, $\sigma$-subadditive mapping of the family of all subsets of $\mathrm{R}^{n}$ into $[0, \infty]$ such that $\mu(A)=$ $\inf \{\mu(B) ; A \subseteq B$ and $B$ Borel set $\}$ and that $\mu(A \cup B)=\mu(A)+\mu(B)$ whenever $\operatorname{dist}(A, B)$ positive (i.e., $\mu$ is a Borel regular metric outer measure). The last property is equivalent to the $\sigma$-additivity of $\mu$ on the family of all Borel sets, see [4], 2.3.2(9).

The support of the measure $\mu$ over $\mathrm{R}^{n}$ is defined by

$$
\operatorname{spt}(\mu)=\left\{x \in \mathrm{R}^{n} ; \mu(B(x, r))>0 \text { for every } r>0\right\} .
$$

Since in our situation we will always have $X=\operatorname{spt}(\mu) \subseteq \mathrm{R}^{n}$, we avoid the use of any special symbol for the space by adopting the following definition.

Definition 0.1. A measure $\mu$ over $\mathrm{R}^{n}$ is said to be uniformly distributed if there is a function $f_{\mu}:(0, \infty) \rightarrow[0, \infty]$ such that

(i) $\mu(B(x, r))=f_{\mu}(r)$ for every $x \in \operatorname{spt}(\mu)$ and $r>0$, and

(ii) $f_{\mu}(r)<\infty$ for some $r>0$.

\section{Uniformly distributed measures in $\mathrm{R}^{n}$}

LeMma 1.1. Let $\mu$ be a uniformly distributed measure over $\mathrm{R}^{n}, x \in \mathrm{R}^{n}$, and $0<s<r<\infty$, then $\mu(B(x, r)) \leq 5^{n}(r / s)^{n} f_{\mu}(s)$.

Proof. Let $Z \subseteq B(x, r)$ be a maximal set such that $\left\|z_{1}-z_{2}\right\|>s / 2$ whenever $z_{1}, z_{2} \in Z$ and $z_{1} \neq z_{2}$. Since the balls $B(z, s / 4), z \in Z$, are mutually disjoint, by comparison of their volumes we infer that $(s / 4)^{n}$ card $Z \leq$ $(5 r / 4)^{n}$. Hence card $Z \leq 5^{n}(r / s)^{n}$ and

$$
\mu(B(x, r)) \leq \sum_{z \in Z} \mu(B(z, s / 2)) \leq f_{\mu}(s) \operatorname{card} Z \leq 5^{n}(r / s)^{n} f_{\mu}(s)
$$

since $\mu(B(z, s / 2))=0$ in case when $B(z, s / 2) \cap \operatorname{spt}(\mu)=\emptyset$ and $\mu(B(z, s / 2))$ $\leq \mu(B(x, s))$ if $x \in B(z, s / 2) \cap \operatorname{spt}(\mu)$.

COROLlaRy 1.2. Let $\mu$ be a uniformly distributed measure over $\mathrm{R}^{n}$. Then

(i) $f_{\mu}(r)<\infty$ for every $r>0$,

(ii) $\operatorname{spt}(\mu)$ is bounded if and only if $\mu\left(\mathrm{R}^{n}\right)<\infty$,

(iii) $\int_{\mathrm{R}^{n}} \exp \left(-s\|z-x\|^{2}\right) d \mu(z)=\int_{0}^{1} \mu(B(x, \sqrt{-(\ln r) / s})) d r$ converges for all $s>0$ and $x \in \mathrm{R}^{n}$, and

(iv) $\int_{\mathrm{R}^{n}} \exp \left(-s\|z-x\|^{2}\right) d \mu(z)=\int_{\mathrm{R}^{n}} \exp \left(-s\|z-y\|^{2}\right) d \mu(z)$ whenever $s>0$ and $x, y \in \operatorname{spt}(\mu)$. 
Proof. Since $f_{\mu}(s)<\infty$ for some $s>0$, (i) follows from Lemma 1.1. If $\operatorname{spt}(\mu)$ is unbounded then there is an infinite set $Z \subseteq \operatorname{spt}(\mu)$ such that $\left\|z_{1}-z_{2}\right\|>2$ whenever $z_{1}, z_{2} \in Z$ and $z_{1} \neq z_{2}$. Since $\mu(B(z, 1))=f_{\mu}(1)>$ 0 for every $z \in Z$, we have $\mu\left(\mathrm{R}^{n}\right)=\infty$.

The formula in (iii) follows from Fubini's theorem (see, e.g., [8], Theorem 1.15), and the finiteness statement follows by using Lemma 1.1 to estimate

$$
\int_{0}^{1} \mu(B(x, \sqrt{-(\ln r) / s})) d r \leq 5^{n} \int_{0}^{1}(-(\ln r) / s)^{n / 2} f_{\mu}(1) d r<\infty .
$$

Finally, (iv) follows from the formula in (iii), since for $x \in \operatorname{spt}(\mu)$,

$$
\int_{0}^{1} \mu(B(x, \sqrt{-(\ln r) / s})) d r=\int_{0}^{1} f_{\mu}(\sqrt{-(\ln r) / s}) d r
$$

does not depend on $x$.

THeOREM 1.3. Let $\mu$ be a uniformly distributed measure over $\mathrm{R}^{n}$ and let $x_{0} \in \operatorname{spt}(\mu)$. For every $x \in \mathrm{R}^{n}$ and $s>0$ let

$$
F(x, s)=\int_{\mathrm{R}^{n}}\left[\exp \left(-s\|z-x\|^{2}\right)-\exp \left(-s\left\|z-x_{0}\right\|^{2}\right)\right] d \mu(z) .
$$

Then

(i) $F(x, s)$ is well-defined and finite for any $x \in \mathrm{R}^{n}$ and any $s>0$; moreover, its definition is independent of the choice of $x_{0} \in \operatorname{spt}(\mu)$.

(ii) $x \in \operatorname{spt}(\mu)$ if and only if $F(x, s)=0$ for every $s>0$, and

(iii) if $x \notin \operatorname{spt}(\mu)$ then there is an $s_{x} \in(0, \infty)$ such that $F(x, s)<0$ for every $s \geq s_{x}$.

Proof. Statement (i) follows from Corollary 1.2, (iii) and (iv). If $x \in \operatorname{spt}(\mu)$ and $s>0$ then $F(x, s)=0$ according to Corollary 1.2, (iv). If $x \notin \operatorname{spt}(\mu)$, let $\varepsilon \in(0,1)$ be such that $B(x, \varepsilon) \cap \operatorname{spt}(\mu)=\emptyset$. Using Lemma 1.1, we estimate

$$
\begin{aligned}
\int_{\mathrm{R}^{n}} \exp \left(-s\|z-x\|^{2}\right) d \mu(z) & =\sum_{k=1}^{\infty} \int_{B(x,(k+1) \varepsilon) \backslash B(x, k \varepsilon)} \exp \left(-s\|z-x\|^{2}\right) d \mu(z) \\
& \leq \sum_{k=1}^{\infty} \exp \left(-s k^{2} \varepsilon^{2}\right) \mu(B(x,(k+1) \varepsilon)) \\
& \leq(10)^{n} \sum_{k=1}^{\infty} \exp \left(-s k^{2} \varepsilon^{2}\right)(k+1)^{n} f_{\mu}(\varepsilon / 2)
\end{aligned}
$$


Hence

$$
\begin{aligned}
\lim _{s \rightarrow \infty} \frac{\int_{\mathrm{R}^{n}} \exp \left(-s\|z-x\|^{2}\right) d \mu(z)}{\int_{\mathrm{R}^{n}} \exp \left(-s\left\|z-x_{0}\right\|^{2}\right) d \mu(z)} \leq \lim _{s \rightarrow \infty} \frac{\int_{\mathrm{R}^{n}} \exp \left(-s\|z-x\|^{2}\right) d \mu(z)}{\exp \left(-s \varepsilon^{2} / 4\right) f_{\mu}(\varepsilon / 2)} \\
\leq \lim _{s \rightarrow \infty}(10)^{n} \sum_{k=1}^{\infty} \exp \left(-s k^{2}\left(\varepsilon^{2}-\varepsilon^{2} / 4\right)\right)(k+1)^{n}=0 .
\end{aligned}
$$

Consequently, there is an $s_{x} \in(0, \infty)$ such that for every $s \geq s_{x}$

$$
\int_{\mathrm{R}^{n}} \exp \left(-s\|z-x\|^{2}\right) d \mu(z)<\frac{1}{2} \int_{\mathrm{R}^{n}} \exp \left(-s\left\|z-x_{0}\right\|^{2}\right) d \mu(z),
$$

which proves (iii) as well as the remaining implication in (ii).

THEOREM 1.4. Let $\mu$ be a uniformly distributed measure over $\mathrm{R}^{n}$. Then

(i) $\operatorname{spt}(\mu)$ is an analytic variety, and

(ii) there is an integer $m \in\{0,1, \ldots, n\}$, a constant $c \in(0, \infty)$ and an open subset $G$ of $\mathrm{R}^{n}$ such that

(1) $G \cap \operatorname{spt}(\mu)$ is an m-dimensional analytic submanifold of $\mathrm{R}^{n}$,

(2) $\mathrm{R}^{n} \backslash G$ is the union of countably many analytic submanifolds of $\mathrm{R}^{n}$ of dimensions less than $m, \mu\left(\mathrm{R}^{n} \backslash G\right)=\mathscr{H}^{m}\left(\mathrm{R}^{n} \backslash G\right)=0$.

(3) $\mu(A)=c \mathscr{H}^{m}(A \cap G \cap \operatorname{spt}(\mu))=c \mathscr{H}^{m}(A \cap \operatorname{spt}(\mu))$ for every subset $A \subseteq \mathrm{R}^{n}$. ( $\mathscr{H}^{m}$ denotes the $m$-dimensional Hausdorff measure.)

Proof. (i) For a direct proof, let $H(x)=\int_{1}^{\infty} \exp \left(-s^{3}\right) F^{2}(x, s) d s$, where $F$ is the function defined in Theorem 1.3. We easily see that $H$ is a welldefined function on $\mathrm{R}^{n}$ and, using Theorem 1.3 (iii) and (iv), that $\operatorname{spt}(\mu)=$ $\left\{x \in \mathrm{R}^{n} ; H(x)=0\right\}$. Moreover, $H$ is an analytic function, since the function $\tilde{H}$ taking at $\left(x_{1}, \ldots, x_{n}\right) \in \mathrm{C}^{n}$ the value

$$
\left.\int_{1}^{\infty} e^{-s^{3}}\left[\int_{\mathrm{R}^{n}}\left(\exp \left(-s \sum_{i=1}^{n}\left(z_{i}-x_{i}\right)^{2}\right)\right)-\exp \left(-s \sum_{i=1}^{n}\left(z_{i}-x_{i}^{0}\right)^{2}\right)\right) d \mu(z)\right]^{2} d s
$$

is well-defined and holomorphic on the whole complex $n$-space $\mathrm{C}^{n}$.

An alternative proof is obtained by noting that, for each $s>0$, the set $\{x: F(x, s)=0\}$ is an analytic variety, and that the intersection of (an arbitrary number of) analytic varieties is again an analytic variety.

(ii) Whenever $U \subseteq \mathrm{R}^{n}$ is an open set and $U \cap \operatorname{spt}(\mu)$ is a nonempty $k$ dimensional analytic submanifold of $\mathrm{R}^{n}$, then the Besicovitch Differentiation Theorem (see [4], 2.9.7) implies that the limit

$$
f(z)=\lim _{r \searrow 0} \mu\left(B(z, r) /\left(\mu+\mathscr{H}^{k}\llcorner\operatorname{spt}(\mu))(B(z, r))\right.\right.
$$


exists at $\left(\mu+\mathscr{H}^{k}\llcorner\operatorname{spt}(\mu))\right.$-almost every $z \in U$ and

$$
\mu(A)=\int_{A} f(z) d\left(\mu+\mathscr{H}^{k}\llcorner\operatorname{spt}(\mu))(z) \text { for any Borel set } A \subseteq U .\right.
$$

(Here $\mathscr{H}^{k} \leftarrow \operatorname{spt}(\mu)(M)=\mathscr{H}^{k}(M \cap \operatorname{spt}(\mu))$ for any set $M$.) Then clearly $f(z)=\lim _{r \searrow 0} f_{\mu}(r) /\left(f_{\mu}(r)+\alpha(k) r^{k}\right)$ for any $z \in U \cap \operatorname{spt}(\mu)$ where $\alpha(k)$ denotes the volume of the $k$-dimensional unit ball. Thus

$$
c=\lim _{r \searrow 0} f_{\mu}(r) /\left(\alpha(k) r^{k}\right)
$$

exists; moreover $c \neq 0$ since otherwise $(*)$ would imply $\mu(U)=0$ and $c \neq \infty$ since otherwise $(*)$ would imply $\mathscr{H}^{k}(U \cap \operatorname{spt}(\mu))=0$. Consequently, $f=c /(c+1)$ at $\left(\mu+\mathscr{H}^{k}\llcorner\operatorname{spt}(\mu))\right.$-almost every point of $U \cap \operatorname{spt}(\mu)$, from which, using $(*)$ once more, we infer that $\mu(A)=c \cdot \mathscr{H}^{k} L \operatorname{spt}(\mu)(A)$ for every Borel set $A \subseteq U$.

Since the statement (ii) is trivial if $\mu$ is the zero measure, we may assume that $\operatorname{spt}(\mu) \neq \emptyset$. Let $G$ be a maximal open subset of $\mathrm{R}^{n}$ such that $G \cap \operatorname{spt}(\mu)$ is an analytic submanifold of $\mathrm{R}^{n}$. Since $G \cap \operatorname{spt}(\mu) \neq \varnothing$ (see [4], 3.4.8), we infer from the above analysis that there is an integer $m \in\{0,1, \ldots, n\}$ such that $0<c=\lim _{r \searrow 0} f_{\mu}(r) /\left(\alpha(m) r^{m}\right)<\infty$. Since this integer $m$ is determined uniquely, we conclude that $G \cap \operatorname{spt}(\mu)$ is an $m$-dimensional analytic submanifold of $\mathrm{R}^{n}$. Therefore, according to [4], 3.4.8, $\mathrm{R}^{n} \backslash G$ is a countable union of analytic submanifolds of $\mathrm{R}^{n}$, all of dimension less than $m$. Hence $\mathscr{H}^{m}\left(\mathrm{R}^{n} \backslash G\right)=0$ and, since $\mu$ is absolutely continuous with respect to $\mathscr{H}^{m}$ (see [4], 2.10.19(1)), $\mu\left(\mathrm{R}^{n} \backslash G\right)=0$. This proves (2). The statement (3) is an easy corollary of (2) and of the analysis at the beginning of the proof of (ii).

THEOREM 1.5. Let $\mu$ be a uniformly distributed measure over $\mathrm{R}^{n}$ with bounded support and let $x_{0} \in \operatorname{spt}(\mu)$. Then $x \in \operatorname{spt}(\mu)$ if and only if

$$
P_{k}(x)=\int_{R^{n}}\left[\langle z-x, z-x\rangle^{k}-\left\langle z-x_{0}, z-x_{0}\right\rangle^{k}\right] d \mu(z)=0
$$

for every natural number $k \geq 1$.

Proof. Because $\operatorname{spt}(\mu)$ is a compact set,

$$
F(x, s)=\sum_{k=1}^{\infty} \frac{(-s)^{k}}{k !} \int_{\mathrm{R}^{n}}\left[\langle z-x, z-x\rangle^{k}-\left\langle z-x_{0}, z-x_{0}\right\rangle^{k}\right] d \mu(z)
$$

and hence $F(x, s)=0$ for every $s>0$ if and only if $P_{k}(x)=0$ for every $k=1,2, \ldots$. 
COROLlaRY 1.6. Let $\mu$ be a uniformly distributed measure over $\mathrm{R}^{n}$ with bounded support. Then this support is an algebraic variety.

Proof. According to Theorem 6, $\operatorname{spt}(\mu)=\bigcap_{k=0}^{\infty}\left\{x \in \mathrm{R}^{n} ; P_{k}(x)=0\right\}$. But each $P_{k}(x)$ is a polynomial in the coordinates of the point $x$. Hence Hilbert's theorem for polynomials over a Noetherian ring (see [1], chap. III, $\$ 2.10$, Corollary 2) implies that there are natural numbers $k_{1}, \ldots, k_{l}$ such that $\operatorname{spt}(\mu)=\bigcap_{j=1}^{l}\left\{x \in \mathrm{R}^{n} ; P_{k_{j}}(x)=0\right\}$. Hence the support of the measure $\mu$ is the zero-set, for example, of the polynomial $P(x)=\sum_{j=1}^{l} P_{k_{j}}^{2}(x)$.

By considering the condition $P_{1}(x)=0$ we obtain the following result which is contained already in [3].

Proposition 1.7. Let $\mu$ be a uniformly distributed measure over $\mathrm{R}^{n}$ with bounded support. Then this support is contained in some $(n-1)$-dimensional sphere.

Proof. $P_{1}(x)=0$ means

$$
\int_{\mathrm{R}^{n}}[\langle x, x\rangle-2\langle z, x\rangle] d \mu(z)=\int_{\mathrm{R}^{n}}\left[\left\langle x_{0}, x_{0}\right\rangle-2\left\langle z, x_{0}\right\rangle\right] d \mu(z) .
$$

We denote by $c=\int_{\mathrm{R}^{n}} z d \mu(z) / \mu\left(\mathrm{R}^{n}\right)$ the barycentr of the measure $\mu$. Then $\langle x, x\rangle-2\langle c, x\rangle=\left\langle x_{0}, x_{0}\right\rangle-2\left\langle c, x_{0}\right\rangle$ and consequently $\|x-c\|^{2}=\left\|x_{0}-c\right\|^{2}$ for any $x \in \operatorname{spt}(\mu)$.

Remark 1.8. If $\operatorname{spt}(\mu)$ is a discrete, bounded set, then it is clearly finite. Let $k$ denote its cardinality. Then

$$
\operatorname{spt}(\mu)=\left\{x \in \mathrm{R}^{n} ; P_{m}(x)=0 \text { for every } m \leq k\right\} .
$$

This can be shown using the Newton identities for the sums of the $m$-th powers.

\section{Uniformly distributed measures in $R$ and $R^{2}$}

Now we investigate uniformly distributed measures on the straight line and on the plane.

Proposition 2.1. The support of a uniformly distributed measure over the straight line $\mathrm{R}$ is either the whole $\mathrm{R}$ or a discrete set.

Proof. It suffices to assume that $\emptyset \neq \operatorname{spt}(\mu) \neq \mathrm{R}$ and to show that $\operatorname{spt}(\mu)$ contains an isolated point. Let $x_{0} \notin \operatorname{spt}(\mu)$. Then there exists some $x_{1} \in \operatorname{spt}(\mu)$ such that $\left|x_{0}-x_{1}\right|=\min \left\{\left|x_{0}-x\right| ; x \in \operatorname{spt}(\mu)\right\}$. We assume $x_{0}<x_{1}$; the case $x_{0}>x_{1}$ is similar. If $x_{1}$ is an accumulation point of $\operatorname{spt}(\mu)$ then there are $x_{2}, x_{3} \in \operatorname{spt}(\mu)$ satisfying $0<2\left(x_{2}-x_{1}\right)<x_{3}-x_{1}<x_{1}-x_{0}$. Hence we can 
choose an $\varepsilon \in\left(x_{3}-x_{2}, x_{3}-x_{1}\right)$. Because $\operatorname{spt}(\mu) \cap\left[x_{0}, x_{1}\right)=\emptyset$, we obtain $\mu\left(B\left(x_{1}, \varepsilon\right)\right)=\mu\left(\left[x_{1}, x_{1}+\varepsilon\right]\right) \leq \mu\left(\left[x_{1}, x_{2}+\varepsilon\right]\right) \leq \mu\left(B\left(x_{2}, \varepsilon\right)\right)$. That implies $\mu\left(\left(x_{1}+\varepsilon, x_{2}+\varepsilon\right)\right)=0$ but $x_{3}$ is an interior point of $\left(x_{1}+\varepsilon, x_{2}+\varepsilon\right)$. This contradiction shows that $x_{1}$ must be an isolated point of $\operatorname{spt}(\mu)$ and hence that $\operatorname{spt}(\mu)$ is a discrete set.

A simple combinatorial proof of the following lemma and of its corollary may be left to the reader.

LEMMA 2.2. Let $\mu$ be a uniformly distributed measure over $\mathrm{R}$ with discrete and unbounded support. Then there are positive reals $a, b$ and a bijection $\phi: \mathrm{Z} \rightarrow \mathrm{R}$ mapping the integers onto $\operatorname{spt}(\mu)$ such that

$$
\phi(i+1)-\phi(i)= \begin{cases}a & \text { for even } i \\ b & \text { for odd } i\end{cases}
$$

COROLlary 2.3. There are three kinds of nonzero uniformly distributed measures over R. The first kind are multiples of the Lebesgue measure by positive constants. The second kind are the unbounded and discrete uniformly distributed measures described in Lemma 2.2. The last kind are bounded and discrete uniformly distributed measures; by Proposition 2.1, such measures may be supported either by a single point or by a pair of points.

The following proposition completely characterizes those uniformly distributed measures over the plane that have a bounded support. A number of examples of such measures with unbounded support can be also constructed, but a general description has not been found yet.

PROPOSITION 2.4. There are three kinds of nonzero uniformly distributed measures over $\mathrm{R}^{2}$ with bounded support.

(i) The support of the measure is a circle.

(ii) The support is formed by the vertices of a regular polygon.

(iii) The support is formed by the vertices of two regular n-gons having the same center and radius.

Proof. Because of Proposition 1.7, we may assume that $\operatorname{spt}(\mu) \subseteq\{x \in$ $\left.\mathrm{R}^{2} ;\|x-c\|=r\right\}$. Let $\phi: \mathrm{R} \rightarrow \mathrm{R}^{2}$ be defined by $\phi(t)=c+(r \cos t, r \sin t)$ and let $g: \mathrm{R} \rightarrow \mathrm{R}$ be such that $g(t)=\mu(\phi(0, t])$ for $t \in[0,2 \pi)$ and $g(t+2 \pi)=g(t)+\mu\left(\mathrm{R}^{2}\right)$ for every $t \in \mathrm{R}$. Then $g$ is the distribution function of some uniformly distributed measure $\lambda$ over $\mathrm{R}$ and $x \in \operatorname{spt}(\lambda)$ if and only if $\phi(x) \in \operatorname{spt}(\mu)$. The statement now follows from Corollary 2.3.

REMARK 2.5. Clearly, a measure over $\mathrm{R}^{n}$ is uniformly distributed provided it is locally finite and fulfills the following homogeneity condition: 
For every pair $x, y \in \operatorname{spt}(\mu)$ there is an isometry $\phi: \mathrm{R}^{n} \rightarrow \mathrm{R}^{n}$ such that $\phi(x)=y$ and $\phi_{\#} \mu=\mu$, where $\left(\phi_{\#} \mu\right)(A)=\mu\left(\phi^{-1}(A)\right)$.

The measures described in Corollary 2.3 and Proposition 2.4 are homogeneous in this sense. On the other hand, the three dimensional measure concentrated on the set

$$
\left\{\left(x_{1}, x_{2}, x_{3}, x_{4}\right) \in \mathrm{R}^{4} ; x_{4}^{2}=x_{1}^{2}+x_{2}^{2}+x_{3}^{2}\right\}
$$

is uniformly distributed (see [5] or [9]) but not homogeneous. It is unknown whether there are non-homogeneous uniformly distributed measures in $R^{2}$ or $\mathrm{R}^{3}$, or in any $\mathrm{R}^{n}$ with bounded support or with discrete support.

\section{An application: Proof of Marstrand's Theorem}

Using our results we will present here an alternative proof of one of the main results in [6] which roughly says that the "density dimension" is always an integer.

THEOREM 3.1. Let $\mu$ be a metric outer measure over $\mathrm{R}^{n}, n \geq 1$, s a nonnegative real number and let the sets $B \subseteq A \subseteq \mathrm{R}^{n}$ satisfy

(i) $\mu(B)>0$, and

(i) $\lim _{r \downarrow 0} r^{-s} \mu(B(x, r) \cap A)$ exists and is positive and finite for any $x \in B$.

Then $s$ is an integer.

Proof. Using standard regularization methods, we infer the measurability of both $x \rightarrow \lim \sup _{r \searrow 0} r^{-s} \mu(B(x, r) \cap A)$ and $x \rightarrow \liminf _{r \searrow 0} r^{-s} \mu(B(x, r)$ $\cap A$ ), and (ii) we may in addition assume that $\tilde{\mu}=\mu\llcorner A$ is Borel regular (i.e., a measure) and locally finite in some open superset $U$ of $A$ and that $B$ is a compact set on which the functions $x \rightarrow k^{s} \tilde{\mu}\left(B\left(x, \frac{1}{k}\right)\right)$ uniformly converge, as $k$ tends to infinity, to a continuous function $d: B \rightarrow(0, \infty)$. The Besicovitch Differentiation theorem ([4], 2.9.7) ensures the existence of an $x_{0} \in B$ with $\lim _{r \backslash 0} \tilde{\mu}\left(B\left(x_{0}, r\right) \backslash B\right) / \tilde{\mu}\left(B\left(x_{0}, r\right)\right)=0$. For $k \geq 1$ we define $\tilde{\mu}_{k}(M)=k^{s} \tilde{\mu}\left(x_{0}+\frac{1}{k} M\right)$ and $B_{k}=k \cdot\left(B-x_{0}\right)$. Since $\lim _{k \rightarrow \infty} \tilde{\mu}_{k}(B(0, i))=$ $d\left(x_{0}\right) i^{s}$ for any integer $i \geq 0$, we infer from well-known weak compactness results for measures, see e.g., [8], Theorem 1.23 or [9], Proposition 1.12, that there is a sequence $k_{i} \nearrow \infty$ of integers and a Radon measure $\Phi$ over $\mathrm{R}^{n}$ such that $\Phi(C) \geq \lim \sup _{i \rightarrow \infty} \tilde{\mu}_{k_{i}}(C)$ for any compact set $C$ and $\Phi(G) \leq$ $\liminf _{i \rightarrow \infty} \tilde{\mu}_{k_{i}}(G)$ for any open set $G$. We show that $\Phi$ is a nonzero uniformly distributed measure with $f_{\Phi}(r)=r^{s} d\left(x_{0}\right)$. Then it easily follows that $s=m$ for the integer $m$ from Theorem 1.4 (ii). This will finish our proof.

Since $\Phi(B(0,1)) \geq d\left(x_{0}\right)>0$, we have $\emptyset \neq \operatorname{spt}(\Phi)$. Let $x \in \operatorname{spt}(\Phi), R>$ 0 and fix a positive $\varepsilon<R$. Then $0<\Phi(B(x, \varepsilon / 2)) \leq \liminf _{i \rightarrow \infty} \tilde{\mu}_{k_{i}}(B(x, \varepsilon))$. According to the choice of $x_{0}$ we have $\lim _{i \rightarrow \infty} \tilde{\mu}_{k_{i}}\left(B(0,\|x\|+2 \varepsilon) \backslash B_{k_{i}}\right)=0$ 
and therefore for any $i$ sufficiently large we may find a point $y_{i} \in B(x, \varepsilon) \cap B_{k_{i}}$. Consequently, we can estimate

$$
\begin{aligned}
\Phi(B(x, R)) & \geq \limsup _{i \rightarrow \infty} \tilde{\mu}_{k_{i}}(B(x, R)) \geq \limsup _{i \rightarrow \infty} \tilde{\mu}_{k_{i}}\left(B\left(y_{i}, R-\varepsilon\right)\right) \\
& =\limsup _{i \rightarrow \infty} k_{i}{ }^{s} \tilde{\mu}\left(B\left(x_{0}+y_{i} / k_{i},(R-\varepsilon) / k_{i}\right)\right) \geq d\left(x_{0}\right)(R-\varepsilon)^{s}
\end{aligned}
$$

since all $\left(x_{0}+y_{i} / k_{i}\right)$ belong to $B$ and the "average density" uniformly converges on $B$. Similarly, one estimates that the $\Phi$-measure of the interior of the ball $B(x, R+\varepsilon)$ is at most $d\left(x_{0}\right)(R+2 \varepsilon)^{s}$ and, tending with $\varepsilon$ to zero, we infer that $\Phi(B(x, R))=d\left(x_{0}\right) R^{s}$ as required.

\section{REFERENCES}

1. Bourbaki, N., Elements de mathématique, Fasc. XXVIII, Algèbre Commmutative, Hermann, Paris 1961.

2. Christensen, J. P. R., On some measures analogous to Haar measures, Math. Scand. 26 (1970), 103-106.

3. Christensen, J. P. R., Uniform measures and spherical harmonics, Math. Scand. 26 (1970), 293-302.

4. Federer, H., Geometric Measure Theory, Grundlehren Math. Wiss. 153 (1969).

5. Kowalski, O. and Preiss, D., Besicovitch-type properties of measures and submanifolds, J. Reine Angew. Math. 379 (1987), 115-151.

6. Marstrand, J. M., The ( $\Phi, s)$-regular subsets of $n$ space, Trans. Amer. Math. Soc. 113 (1964), 369-392.

7. Mattila, P., Differentiation of measures in uniform spaces, Lecture Notes in Math. 794 (1979).

8. Mattila, P., Geometry of Sets and Measures in Euclidean Spaces, Cambridge University Press 1995.

9. Preiss, D., Geometry of measures in $\mathrm{R}^{n}$ : Distribution, rectifiability and densities, Ann. of Math. 125 (1987), 537-643.

10. Studený, M., Differentiation of measures in metric spaces, Thesis, Prague 1981

ÚAM MFF UK

MLYNSKÁ DOLINA

84215 BRATISLAVA

SLOVAKIA

DEPARTMENT OF MATHEMATICS

UNIVERSITY COLLEGE LONDON

GOWER STREET

LONDON WC1E 6BT

UK
CURRENT ADDRESS:

MAX-PLANCK-INSTITUTE FÜR MATHEMATIK IN DEN NATURWISSENSCHAFTEN

INSELSTRAßE 22-26

D-04103 LEIPZIG

GERMANY 